\title{
Migraine can be Managed with Virtual Scanning: Case Report
}

\author{
E. Ewing ${ }^{1}$, G. Ewing ${ }^{1, *}$ and E.U. Nwose ${ }^{2}$
}

\author{
${ }^{1}$ Montague Healthcare, Mulberry House, 6 Vine farm Close - Cotgrave, Nottinghamshire NG12 $3 T U$ England and \\ ${ }^{2}$ Institute of Clinical Pathology \& Medical Research, South West Pathology Service, Smollett Street Albury NSW 2640 \\ Australia
}

\begin{abstract}
It is known that Virtual Scanning is a cognitive technology that translates electro-physiology arising from biophotons emission into clinical information. The technology promises diagnostic and therapeutic applicability for all categories of migraine. We present a case of migraine in which the patient testifies that the technology helped to alleviate the physiological symptoms. The biochemical basis for anti-platelet medication vis-à-vis stasis and thrombogenesis in migraine and the technology is highlighted. The validity of the clinical benefits of light therapy and Virtual Scanning technology in particular, which can be employed as an emerging non-invasive option to manage migraine, requires a consideration and research in order to develop a framework for integration into conventional medical practice.
\end{abstract}

\section{INTRODUCTION}

Virtual Scanning (VS) technology is an emerging concept in alternative clinical diagnosis and therapy. It originated from Russia. The technology is practically a computer programming of cognate function of the brain to translate color cognition in order to evaluate specific influences from different parts of the nervous system $[1,2]$.

Claude Bernard had conceptualized that the internal environment of organism vis-à-vis physiological state is constant with the existence of control processes to achieve such constancy, and further envisaged a possible mathematical modeling applications [3]. The mathematical modelling of the body's relationship with its environment was developed in Russia $[2,4]$.

It casts new light upon how the brain processes light emissions arising from biochemical signals and relates same to physiological states. It may be likened to other emissionbased technologies such as x-rays, positron-emission, magnetic resonance, ultrasound, but there is a difference. Other technologies involve the body's interaction with, and response to, some exogenous agents being inputted to the system as source of emission.

Virtual Scanning is based upon the body's inherent biochemical processes as source of light emission and color defects. The practical diagnostic methodology includes interviewing the patient, followed by the patient using the computer system to solve a standard task that involves memorization of color and answering questions thereafter. The computer then processes the information and shows the results on the screen [4].

Each condition comprises a pairing of blue and red signals. The red signals report the extent of the pathology whilst the blue signal reports the extent of the body's natural

*Address correspondence to this author at the Montague Healthcare, Mulberry House, 6 Vine farm Close - Cotgrave, Nottinghamshire NG12 3TU England; E-mail: graham.ewing@montague-diagnostics.co.uk compensatory response. The scale and balance of pathology and compensation enables the practitioner or researcher to interpret and apply results [5].

What has not been reported is whether the technology can be used to manage clinical cases of migraine. Furthermore, the metabolic basis of conditions that can be diagnosed and treated with the technology has also yet to be discussed. We present a case of migraine in which the patient testifies that the technology helped to alleviate. We postulate the oxidative stress concept as the metabolic basis.

\section{CASE PRESENTATION}

A 59 years old female diagnosed with migraine since 11 years of age. She was also variously diagnosed depression, rheumatism and spondylosis and in 1996 underwent a single mastectomy for breast cancer. For the migraines, she has been treated with many prescription drugs over the last 4050 years, including amongst others, Almagram, which did help at first but became less effective over time. She was also treated for arthritis with ibuprofen initially and finally with paracetamol. The migraine severity got worse in August 2004. The GP's final suggestion was that she needed somewhere quieter than the hospital ward; that she should go into a dark room, sleep and rest for the rest of the weekend.

The option of Virtual Scanning therapy was advised and considered. An appointment was made for 31-08-2004. Since starting the therapy there has been a complete cessation of migraines. Although, there had been few occasional headaches, it's been a great relief. The aches and pains arising from arthritis, rheumatism and spondylosis are also greatly improved.

\section{DISCUSSION}

There have been interests on the potential of chromotherapy in migraine management. It has been reported that people with visual stress without headaches, relative to those with symptoms of migraine or severe headaches, showed the 
largest amplitude visual evoked potential response in the no lens condition; whereas the reverse is the case when wearing prescribed lens [6]. Chromotherapy dates from the ancient Egypt to the contemporary treatment of seasonal affective disorder. It is one of the concepts of irradiation of acupuncture points in complementary medicine [7]. This case report indicates a potential for an emerging technology for migraine management in clinical practice.

The diagnostics on the VS always ends with a determination of patient treatment strategy, which may include recommendation to support optimum cellular composition and/or glucose content of blood and ensuring the optimum amount of circulating blood amongst others [4]. Perhaps, the question would be: how generalize the technology to manage clinical cases of migraine? The answer to this question is the objective of the case report. It is pertinent to identify the gap in knowledge between what is already known and what yet unknown about migraine with regards to light therapy.

\section{Facts About Migraine in Relation to Light Therapy}

Biochemical and physiological processes enable the generation of reactive oxygen species (ROS) and consequential existence of oxidative stress. This involves the brain and is implicated in migraine conditions [8]. The feedback and feedforward responses to oxidative stress reactions, which are part of the development of pathological processes and systemic instability, involve antioxidants in a proteinsubstrate manner and ultra-weak light emission. The latter is perceived and memorized as characteristic of different disease conditions according to color and intensity. That is, the emission is a measurable luminescent effect that is different for different diseases [2, 4, 9].

Furthermore, anti-platelet medication for treatment of migraine is a traditional medical practice and clopidogrel is currently being advocated as useful for migraine treatment in conventional practice $[10,11]$. To the inquisitive mind, this raises the question regarding how anti-platelet medication vis-à-vis stasis and thrombogenesis may be related to the present topics of migraine and the technology.

However, the mechanism of chromotherapy has yet to be explained. The biochemical basis of diseases that can be diagnosed and/or treated with light therapy has also yet to be elucidated. Hence VS technology is still limited to alternative and complementary medicine practice.

\section{The Un-Articulated Facts About Migraine in Relation to Light Therapy i.e. the Gap in Knowledge:}

It is thinkable that Virtual Scanning as a computer based cognitive technology is able to treat migraine by the principle of biofeedback, cognitive or mind-body therapy $[12,13]$. It is therefore necessary to note some un-articulated facts

1. Migraine is a system disease [14]. Several key factors such as blood viscosity affect cerebral blood flow, which is associated with migraine $[15,16]$.

2. ROS are generated during glucose metabolism and affects blood viscosity [17]. ROS and their reactant amino acids are biochemical components of the body's normal physiology, and are major factors in biofeedback [18].
3. The rate of glucose metabolism is directly related ROS generation and by default related to risk of stasis and thrombogenesis. Hence the conventional use of aspirins as intervention therapy in diabetes.

4. The level of blood glucose is a factor in VS technology [4]. Oxidative stress activity, which is influenced by glucose level/metabolism, involves antioxidants reaction with protein-substrate and ultra-weak light emission that would be characteristic of different disease conditions differentiated by color and intensity $[2,9]$.

5. The processes of biofeedback are involuntary, subconscious and involve complex neurological changes including the scenario whereby cerebral blood flow enhances the release of ROS that react with nitric oxide, which otherwise maintains a dilated and/or relaxed blood vessel and pressure [8].

6. Virtual Scanning treats migraines which arise due to stress, lack of blood volume (e.g. following pregnancy or at the end of menstrual periods) or low blood pressure. These are systemic effects. It cannot treat migraines where the cause is due to physical damage such as arising due to car accidents or falls [5].

What is yet to be appreciated is whether determination and management of biophoton emissions from oxidative stress can be employed in the clinical management of migraine. What this report adds to knowledge is the potential for VS technology to bridge the gap. That is, this report postulates the concept of oxidative stress, which has a measurable bioluminescent effect, as the biochemical basis in migraine that underpins the non-pharmacological biofeedback and/or cognitive management principle.

This report aligns the biochemistry and concept of oxidative stress in conventional practice with naturaceutical therapy in complementary medicine. This postulation is supported by the fact that the patient's GP had earlier suggested relaxation in a place that is quieter than a hospital ward. That is, the GP had intended adopting one of the principles of mind-body intervention before the patient turned to VS therapy.

Only one case presentation is limited to cover the technology. Therefore, we acknowledge that while this case report presents a novel clinical observation, our postulation is subject to further studies to advance the understanding.

\section{CONCLUSION}

This case report provides support for the use of Virtual Scanning as a diagnostic and cognitive therapy for migraine. In particular, it provides an explanation aligning the technology with the principles of mind-body intervention in complementary and the principles of both hyperglycaemiainduced oxidative stress and systems physiology in conventional medicine.

\section{COMPETING INTEREST}

Dr. Elena Ewing is a complementary medicine practitioner and together with Mr. Graham Ewing co-owns Montague Healthcare. Dr. Uba Nwose has no competing interest to declare. 


\section{ACKNOWLEDGEMENT}

The 'case presentation' section was paraphrased from the patient's written perspective provided to Dr Elena Ewing.

\section{REFERENCES}

[1] Ewing G, Ewing E, Hankey A. Virtual scanning--a new system of medical assessment and treatment: Part I. Assessment. J Altern Complement Med 2007; 13(2): 271-85.

[2] Hankey A, Ewing E. New Light on Chromotherapy: Grakov's 'Virtual Scanning' System of Medical Assessment and Treatment. Evid Based Complement Alternat Med 2007; 4(2): 139-44.

[3] Noble D. Claude Bernard, the first systems biologist, and the future of physiology.' Exp Physiol 2008; 93(1): 16-26

[4] International University of Fundamental Studies. Virtual Scanning Technology "Strannik". [Web Page] 2006. Available online at: http://www.iufs.edu/strannik.ru/. Accessed $28^{\text {th }}$ January 2009.

[5] Ewing.G.W. and Ewing E.N. Virtual Scanning a new generation of medical technology beyond biomedicine? Pub Montague Healthcare books: 2007.

[6] Riddle PM, Wilkins A, Hainline L. The effect of colored lenses on the visual evoked response in children with visual stress. Optom Vis Sci 2006; 83(5): 299-305.

[7] Cocilovo A. Colored light therapy: overview of its history, theory, recent developments and clinical applications combined with acupuncture. Am J Acupunct 1999; 27(1-2): 71-83.

[8] Ciancarelli I, Tozzi-Ciancarelli MG, Spacca G, Di Massimo C, Carolei A. Relationship between biofeedback and oxidative stress in patients with chronic migraine. Cephalalgia 2007; 27(10): 113641.
[9] Rummel' NN, Kratasyuk VA, Maznyak OM, Inzhevatkin EV, Nefedov VP. Bioluminescent analysis of intensity of pathological oxidative processes in cells of perfused rat liver after hyperthermia. Bull Exp Biol Med 2003; 135(1): 43-5.

[10] Rogers KL, Grice ID, Griffiths LR. Inhibition of platelet aggregation and 5-HT release by extracts of Australian plants used traditionally as headache treatments. Eur J Pharm Sci 2000; 9(4): 35563.

[11] Lacroix JM, Clarke MA, Bock JC, Doxey N, Wood A, Lavis S. Biofeedback and relaxation in the treatment of migraine headaches: comparative effectiveness and physiological correlates. J Neurol Neurosurg Psychiatry 1983; 46(6): 525-32.

[12] Lawden MC, Cleland PG. Achromatopsia in the aura of migraine. J Neurol Neurosurg Psychiatry 1993; 56(6): 708-9.

[13] Astin JA, Shapiro SL, Eisenberg DM, Forys KL. Mind-Body Medicine: State of the Science, Implications for Practice. J Am Board Fam Pract 2003; 16(2): 131-47.

[14] Vanmolkot FH, Van Bortel LM, de Hoon JN. Altered arterial function in migraine of recent onset. Neurology 2007; 68(19): 1563-70.

[15] Hanington E. Migraine: the platelet hypothesis after 10 years. Biomed Pharmacother 1989; 43(10): 719-26.

[16] Meyer JS, Terayama Y, Konno S, Margishvili GM, Akiyama H, Rauch RA, Mortel KF, Wills PM. Age-related cerebrovascular disease alters the symptomatic course of migraine. Cephalalgia 1998; 18(4): 202-8; discussion 171.

[17] Nwose EU, Richards RS, Kerr RG, Tinley R, Jelinek H. Oxidative damage indices for the assessment of subclinical diabetic macrovascular complications. Br J Biomed Sc 2008; 65(3): 136-41.

[18] Camara AK, Riess ML, Kevin LG, Novalija E, Stowe DF. Hypothermia augments reactive oxygen species detected in the guinea pig isolated perfused heart. Am J Physiol Heart Circ Physiol 2004; 286(4): H1289-99. 